Instability, Chaos and Predictability in Celestial Mechanics and

Stellar Dynamics
Copyright 1992 by Nova Science Publishers, Inc. All rights of reproduction in any form reserved.

ISBN 1-56072-054-9

\title{
Planar Oscillation of the Satellite Near PARAMETRIC RESONANCE IN THE ELLIPTIC ORBIT
}

\author{
Ram Binoy Singh \\ Department of Mathematics, University of Bihar, Muzaffarpur
}

\begin{abstract}
ARSTRACT
This paper is devoted to the study of motion of an artificial satellite relative to its centre of mass near parametric resonance in eliiptic orbit. It is well known fact that the satellite, of the form of an ellipsoid with three unequal axes, while moving about the central planet, oscillates about the stable position of equilibrium ( $t$ he longest axis of the satellite coinciding with the radius vector of its centre of mass). The oscillation of the satellite about this position of equilibrium in the orbital plane of its centre of mass is described by a well known second order nonlinear differential equation with a periodic sine force. Naturally there will be resonance cases (main as well as parametric) for such a systems. In the previous author's work [5], it was discovered a series of parametric resonances for the system whiqh corresponds to $n=\frac{1}{2} k$ where $k$ is a non-zero integer and $n$ is a parameter depending on the shape of the satellite. The parametric resonance, for $k=1$, has been considered here. The first approximate solution of the equation of motion has been obtained by Eogoliubov-Krilov method with e (the eccentricity of the orbit of the centre of mass of the satellite) as the small parameter. This method enables us to visualise the oscillation of the satellite for the resonance case as well as near the resonance. Three stationary values of the amplitudes and phase of oscillation have been obtained, out of which only one is stable near this particular parametric resonance. At the resonance there appear only one stationary regime of oscillation with a very small amplitude.
\end{abstract}




\section{INTRODUCTION}

Consider the motion of an artificial satellite in the form of an ellipsoid with three unequal axes relative to the centre of mass which moves along a Keplerian elliptic orbit. The satellite, while moving about the central planet,oscillates about some position of equilibrium on account of the presence of the gravitational moment. The planar oscillatory motions of the satellite is described by the well known non1 inear second order differential equation [1]

$(1+e \cos v) \frac{d^{2} \delta}{d v^{2}}-2 e \sin v \frac{d \delta}{d v}+n^{2} \sin \delta=4 e \sin v$

where $n^{2}=3(A-C) / B$.

$B$ is the moment of inertia of the satellite with respect to the axis perpendicular to the orbital plane of its centre of mass $A$ and $C$ being the moments of inertia about principal axes 1 ying in the orbital plane and such that $B>A>C$. Also $\delta=2 \psi$ where $\psi$ is the angle which the radius vector of the centre of mass of the satellite relative to the central force makes with its longest axis (Fig. 1). v and e are the true anomaly of the centre of mass of the satellite and the eccentricity of its orbit respectively.

When the centre of mass of the satellite moves along a circular orbit $(e=0)$, and hence two positions of equilibrium can be easily obtained as $\delta=0$ and $\delta=\pi$. The first equilibrium position $\psi \delta=0$ (i.e. $\psi=0$ ) is stable and it corresponds to the satellite's position in which the longest axis of inertia coincides with the radius vector of the centre of mass (Fig. 1).

The system moves under a forced vibration on account of the right hand periodic sine force in the equation (1.1). The characteristic feature of the oscillatory systen described by the equation of the type (1.1) is the occurrance of resonance (main as well as parametric). The parametrid rebonance were earlier found for $n= \pm 1 / 2[1]$ and $n=9 / 4[4]$. Al so the author [5] has discovered a series of parametric resonances for $n=1 / 2 k$ where $k$ is a non-zero integer.

The case $n=1 / 2$ was considered by Beletsky,v.V. but he did not elaborate the behaviour of the motion at and near the resonance. The solution of the problem in the general case which is valid at the resonance as well as near the resonance, has been attempted by Eogoliubov-Krilov method for very small value of the eccentricity.

\section{SOLUTION OF THE EQUATION OF PLANAR OSCILLATION NEAR RE- SONANCE}

Consider the eccentricity e, a small quantity of the first order infinitesinal and assume that the non-linearity of 
the equation can also produce.the effect of the order of the eccentricity.

The equation of motion (1.1) can now be written as

$$
\frac{\mathrm{d}^{2} \delta}{d v^{2}}+\mathrm{n}^{2} \delta=e\left[4 \sin \mathrm{v}+2 \delta^{\prime} \sin \mathrm{v}-\delta^{\prime \prime} \cos \mathrm{v}+\alpha(\delta-\sin \delta)\right]
$$

where $d=\frac{\dot{n}^{2}}{e}$.

The solution in the first approximation of (2.1) at the resonance $n=\frac{1}{2}$ will be sought in the form:

$$
\delta=a \cos \phi, \phi=\frac{1}{2} v+k
$$

where amplitude a and phase $k$ must satisfy the system of ordinary differential equations.

$$
\begin{aligned}
& \frac{d a}{d v}=e A_{1}(a, k) \\
& \frac{d k}{d v}=n-\frac{1}{2}+e B_{1}(a, k)
\end{aligned}
$$

and $A_{1}, B_{1}$ are the particular solution and periodic with resrespect to $k$ of the system of partial differential equations:

$$
\begin{aligned}
\left(n-\frac{1}{2}\right) \frac{\partial A_{1}}{\partial k}-2 a n B_{1} & =\frac{1}{2 \pi^{2}} \sum_{\sigma=\infty}^{-\infty} e^{i 2 \sigma \theta} \\
& \times \int_{0}^{2 \pi} \int_{0}^{2 \pi} f_{0}(a, v, \phi) e^{-i 2 \sigma \theta^{\prime}} \cos \phi d v d \phi \\
a\left(n-\frac{1}{2}\right) \frac{\partial B_{1}}{\partial k}+2 n A_{1} & =-\frac{1}{2 \pi^{2}} \sum_{\sigma=\infty}^{-\infty} e^{i 2 \sigma \theta}|1| \frac{1}{2 \pi} \int_{0}^{2 \pi} f_{0}(a, b, \phi) e^{i 2 \sigma \theta^{\prime}} \sin \phi d v d \phi
\end{aligned}
$$

where $\theta=\phi-\frac{v}{2}=\theta^{\prime}$

$$
\begin{aligned}
f_{0}(a, v, \phi)= & 4 \sin v-2 \text { an } \sin v \sin \phi+a n^{2} \cos v \cos \phi+ \\
& +\alpha[a \cos \phi-\sin (a \cos \phi)]
\end{aligned}
$$


Substituting the value of $f_{0}(a, v, \phi)$ of the right hand side of (2.4) and then integrating, we obtain

$$
\begin{aligned}
& \left(n-\frac{1}{2}\right) \frac{\partial A_{1}}{\partial k}-2 a n B_{1}=\alpha\left(a-2 J_{1}(a)\right)+\frac{a n(n-2)}{2} \cos 2 k \\
& a\left(n-\frac{1}{2}\right) \frac{\partial B_{1}}{\partial k}+2 n A_{1}=-\frac{a n(n-2)}{2} \sin 2 k
\end{aligned}
$$

The particular solution, periodic with respect to $k$ of this system is easily obtained as

$$
\begin{aligned}
& A_{1}=-\frac{a n(n-2)}{2} \sin 2 k \\
& B_{1}=-\frac{\alpha}{2 a n}\left[a-2 J_{1}(a)\right]-\frac{n(n-2)}{2} \cos 2 k
\end{aligned}
$$

where $J_{1}$ (a) is the Bessel's function of the first order. Substituting values of $A_{1}$ and $B_{1}$ from (2.6) in (2.3), we get:

$$
\begin{aligned}
& \frac{d a}{d v}=-\frac{\operatorname{ean}(n-2)}{2} \sin 2 k \\
& \frac{d k}{d v}=\frac{n}{2}-\frac{1}{2}+\frac{n J_{1}(a)}{a}-\frac{e n(n-2)}{2} \cos 2 k
\end{aligned}
$$

The system of equations (2.7) can be written as

$$
\begin{aligned}
& \frac{\mathrm{da}}{\mathrm{dv}}=-\frac{1}{\mathrm{a}} \cdot \frac{\partial \mathrm{E}}{\partial \mathrm{k}} \\
& \frac{\mathrm{dk}}{\mathrm{dv}}=\frac{1}{\mathrm{a}} \frac{\partial \mathrm{H}}{\partial \mathrm{a}}
\end{aligned}
$$

where

$$
H=-\frac{e n(n-2)}{4} a^{2} \cos 2 k+n\left[\frac{a^{2}}{4}-\left(J_{0}(a)-1\right)\right]-\frac{a^{2}}{4}
$$

Here $J_{0}(a)$ is the Bessel's function of zero order. Obviously the system of equations (2.8) has a first integral of the form

$$
\mathbf{H}=\mathrm{C}_{\mathrm{o}}
$$

which reduces the problem to quadrature. Here $\mathrm{C}_{0}$ is the constant of integration. 
Fowever, it is preferable to analyse the integral curves in the phase plane $(a, k)$. In order to plot the integral curves, let us put the equation (2.10) in the form:

$$
-a^{2} e n(n-2) \cos 2 k=4 C_{0}+a^{2}(1-2 n)+4 n f(a)
$$

where

$$
f(a)=J_{0}(a)+\frac{a^{2}}{4}-1=\frac{a^{4}}{2^{6}}-\ldots
$$

The integral curves (2.11) have been plotted in (Fig. 2) for $n=0.55$ and $e=0.01$. Clearly there exist $t$ hree stationary regime of the amplitude for $k=0$ and $k= \pm \frac{\pi}{2}$ out of which onl $y$ one is stable for $k=0$.

The stationary regimes of the amplitude and phase are given by equating the right hand side of equations (2.7) to zero:

$$
\frac{e a n(n-2)}{2} \sin 2 k=0
$$

i.e.

$$
\frac{n}{2}-\frac{1}{2}+\frac{n J_{1}(a)}{a}-\frac{\text { en }(n-2)}{2}-\cos 2 k=0 .
$$

The first equation gives $k=0, \pm \frac{\pi}{2}, \ldots$

The second equation of (2.13) can be put in the form

$$
\frac{a^{4}}{3 \times 2^{7}}-\frac{a^{2}}{2^{4}}=\frac{1}{2 n}-1 \pm \frac{e(n-2)}{2}
$$

where plus and minus signs corresponds to the values $k=0$ and $k= \pm \frac{\pi}{2}$ respectivel $y$ and in the Bessel's lfundtion onl $y$ first three terms of the expansion has been retained.

The stationary values of the amplitude for $n=0.55$ and $k=0$ has been obtained as $a=1.25$ and for $n=0.55$ and $k= \pm \frac{\pi}{2}$ they are $a=1.15$. Onl $y$ one of $t$ hese stationary $s o-$ lutions ( $i . e, a=1.25$ and $k=0$ ) is stable which is obvious from (Fig. 2). As $n$ decreases from 0.55 to 0.5 , the stable stationary value of the amplitude reduces to $a=0.34, k=0$ and the other two unstable stationary solutions vanishes. As the value of $n$ decreases further there will be no stationary value of the amplitude. The system will be moving with changing amplitude and phase. However, from this nature of the integral curves in (Fig. 2), it follows that the amplitude 

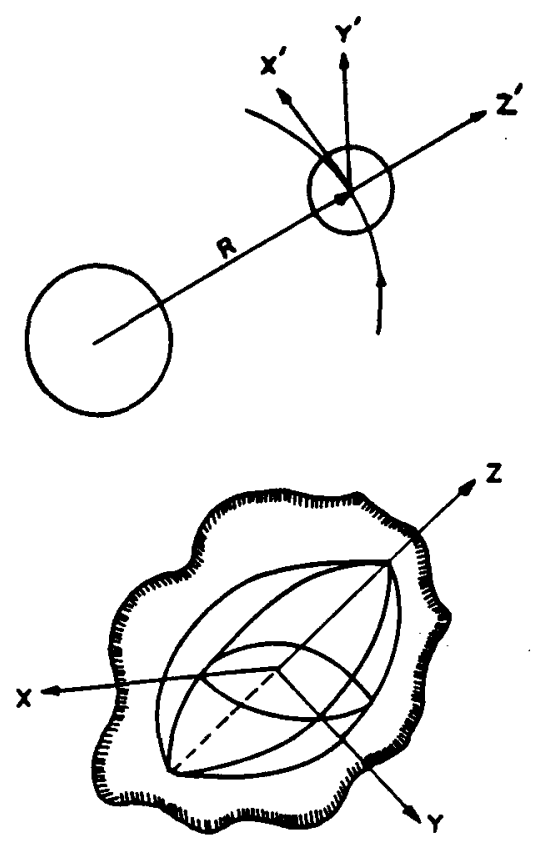

$B>A>C$

FIG. 1

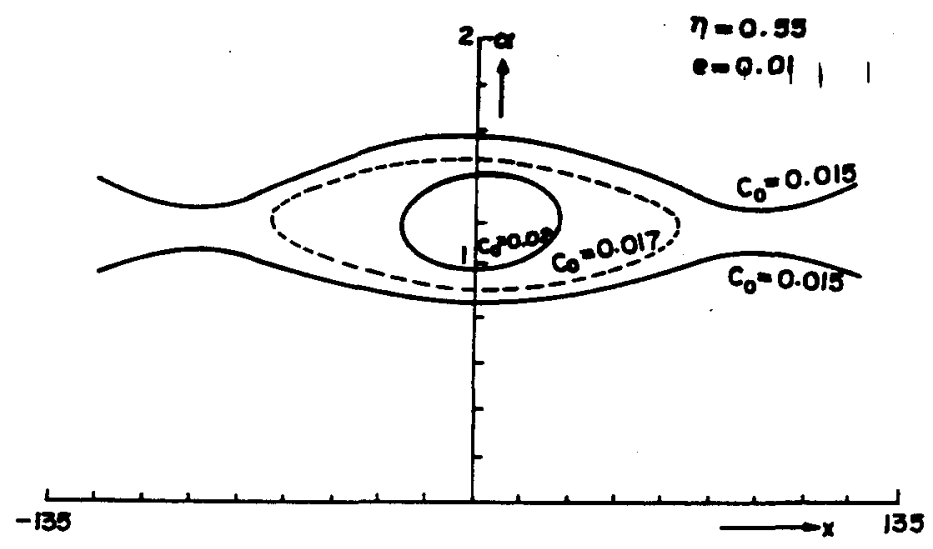

FIG. 2

OSCILLATION OF THE SATELLITE IN ELLIPTIC ORBIT. AMPLITUDE PHASE CHARACTERISTIC FOR $\eta=0.55, e=0.01$ 


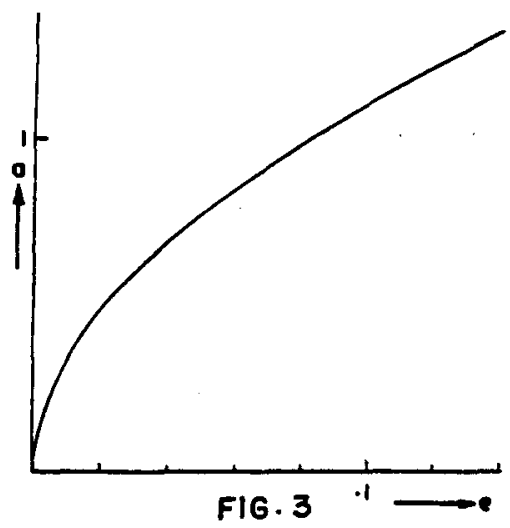

MAXIMUM AMPLITUDE OF OSCILLATION IN ELLIPTIC ORBIT FOR PARAMETRIC RESONANCE $\eta=1 / 2, K=0$

will never increase indefinitely. It will just oscillate between two finite values for different $k$.

The expression for the amplitude of oscillation in the resonance case is given by substituting $n=\frac{1}{2}$ in (2.14) and is obtained as:

$$
a=2 \sqrt{3}(1-\sqrt{1-2 \bar{e}})
$$

The (Fig.3) show the maximum value of the stationary amplitude for different values of $e$ in the resonance case which has been plotted with the help of (2.15). The amplitude increases with increasing $e$. As the formula (2.15) has been obtained from the first approximation, it will give better result only for the small values of $e$.

\section{REF ERENCES}

[1] Belatsky, V.V.(1965), Motion of Artificial Satellite Relative to its Centre of Mass (Russian), Nauka, Moscow.

[2] Bogoliubov, N.N. (1971), Asymptotic Methods in the Theory of Non-1inear Oscillations (Gordon and Breach, New York).

[3] Chernousko, F.L. (1963), Resonance Phenomena in the Yotion of Satellite Relative to its Centre of Mass, Zhur nal vichislitel noi Mathematiki and Mathematicheskoi physiki, Vol. 3, No .3, pp. $528-538$.

[4] Jlataustov, B.A., Okhotsimsky, D.E., Sareechev, B.A., and Torzhevsky, A.P. (1964), Periodic Solution of the Problem of Planar Oscillation of the Satellite in Elliptic Orbit, Kosnicheskie Issledovania, Vol. 2, No.5,pp. $658-666$.

[5] Singh, R.E. and Demin,V.G. (1973), Nonlinear Planar Oscillation of a Solid Body in Flliptic Orbit, Kosmicheskie Issledovania, Vol. 11, No.2. 